University of Texas Rio Grande Valley

ScholarWorks @UTRGV

Chemistry Faculty Publications and

Presentations

College of Sciences

$4-22-2014$

\title{
Communication: Conical Intersections Between Vibrationally Adiabatic Surfaces in Methanol
}

Mahesh B. Dawadi

The University of Texas Rio Grande Valley, mahesh.dawadi@utrgv.edu

David S. Perry

Follow this and additional works at: https://scholarworks.utrgv.edu/chem_fac

Part of the Chemistry Commons

\section{Recommended Citation}

Dawadi, M. B., \& Perry, D. S. (2014). Communication: Conical intersections between vibrationally adiabatic surfaces in methanol. The Journal of Chemical Physics, 140(16), 161101. https://doi.org/10.1063/ 1.4871657

This Article is brought to you for free and open access by the College of Sciences at ScholarWorks @ UTRGV. It has been accepted for inclusion in Chemistry Faculty Publications and Presentations by an authorized administrator of ScholarWorks@ UTRGV. For more information, please contact justin.white@utrgv.edu,william.flores01@utrgv.edu. 


\section{Communication: Conical intersections between vibrationally adiabatic surfaces in methanol}

Cite as: J. Chem. Phys. 140, 161101 (2014); https://doi.org/10.1063/1.4871657

Submitted: 05 March 2014 . Accepted: 31 March 2014 . Published Online: 22 April 2014

Mahesh B. Dawadi, and David S. Perry

\section{ARTICLES YOU MAY BE INTERESTED IN}

Trajectory Surface Hopping Approach to Nonadiabatic Molecular Collisions: The Reaction of $\mathrm{H}^{+}$with $\mathrm{D}_{2}$

The Journal of Chemical Physics 55, 562 (1971); https://doi.org/10.1063/1.1675788

An extended $E \otimes e$ Jahn-Teller Hamiltonian for large-amplitude motion: Application to vibrational conical intersections in $\mathrm{CH}_{3} \mathrm{SH}$ and $\mathrm{CH}_{3} \mathrm{OH}$

The Journal of Chemical Physics 147, 044306 (2017); https://doi.org/10.1063/1.4994699

Molecular dynamics with electronic transitions

The Journal of Chemical Physics 93, 1061 (1990); https://doi.org/10.1063/1.459170

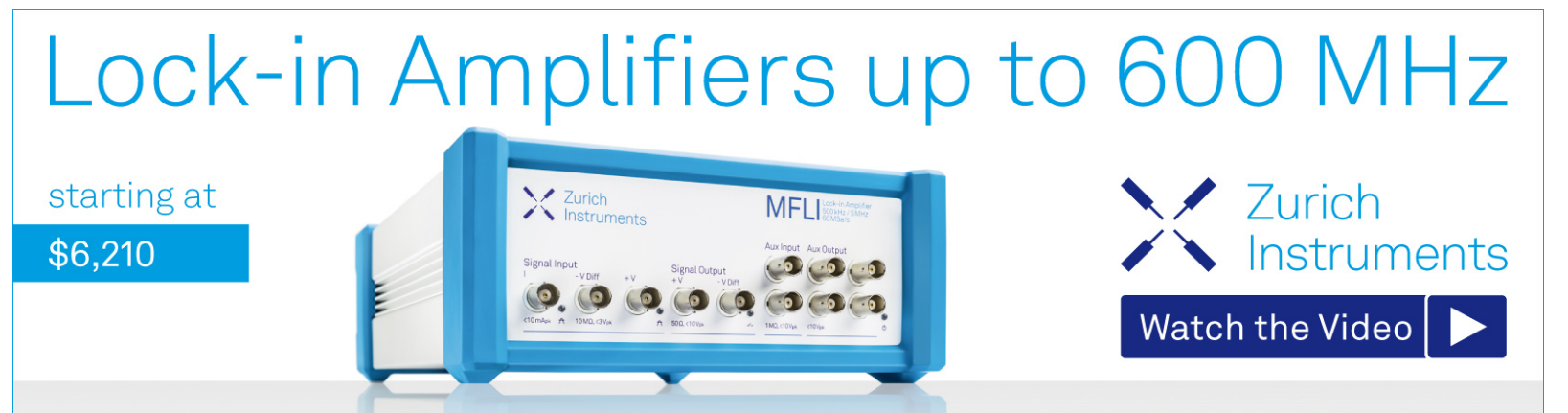

J. Chem. Phys. 140, 161101 (2014); https://doi.org/10.1063/1.4871657

140, 161101

(c) 2014 AIP Publishing LLC. 


\title{
Communication: Conical intersections between vibrationally adiabatic surfaces in methanol
}

\author{
Mahesh B. Dawadi and David S. Perry a) \\ Department of Chemistry, The University of Akron, Akron, Ohio 44325-3601, USA
}

(Received 5 March 2014; accepted 31 March 2014; published online 22 April 2014)

\begin{abstract}
A set of seven conical intersections (CI's) in methanol between vibrationally adiabatic surfaces is reported. The intersecting surfaces represent the energies of the two asymmetric $\mathrm{CH}$ stretch vibrations regarded as adiabatic functions of the torsion and $\mathrm{COH}$ bend angles. The ab initio data are well described by an extended Zwanziger and Grant $(E \otimes e)$ model [J. W. Zwanziger and E. R. Grant, J. Chem. Phys. 87, 2954 (1987)] that might also be regarded as an extension of the XHL model [L.-H. Xu, J. T. Hougen, and R. M. Lees, J. Mol. Spectrosc. 293-294, 38 (2013)]. The CI's illuminate the role of geometric phase in methanol. More generally, they suggest the importance of energy transfer processes localized near the CI's. () 2014 AIP Publishing LLC. [http://dx.doi.org/10.1063/1.4871657]
\end{abstract}

Conical intersections (CI's) between electronic potential energy surfaces are now known to be widespread throughout electronic spectroscopy and are responsible for ultrafast electronic relaxation in diverse circumstances. ${ }^{1-3}$ Whereas these electronic surfaces represent the adiabatic separation of electronic and nuclear motions under the Born-Oppenheimer approximation, it is also possible in some cases to make an approximate adiabatic separation of fast and slow vibrational motions. In such cases, the motion of the high-frequency vibrations, which might include hydride stretches, can be solved quantum mechanically at each molecular geometry along the low frequency, large-amplitude torsional or bending coordinates. These slower motions can then be solved in an effective potential that is the sum of the electronic potential plus the high-frequency vibrational energies as they vary in the largeamplitude space. Recently, Hamm and Stock ${ }^{4,5}$ introduced the concept of CI's between vibrationally adiabatic surfaces as a source of ultrafast vibrational relaxation.

The adiabatic approximation has been applied to methanol, ${ }^{6-8}$ the high frequency vibrations being the $\mathrm{OH}$ and $\mathrm{CH}$ stretches $\left(3860,2999,2956\right.$, and $\left.2844 \mathrm{~cm}^{-1}\right)$ and the low frequency coordinate being the torsional angle, $\gamma$. Fehrensen et $a .^{7}$ applied an adiabatic reaction path Hamiltonian to account for both the decrease of the torsional tunneling splittings with $\mathrm{OH}$ stretch $\left(v_{1}\right)$ excitation and also the inverted torsional tunneling splittings in the first excited states of the asymmetric $\mathrm{CH}$ stretches, $v_{2}$ and $v_{9}$. They found that a geometric phase of -1 was accumulated for the $\mathrm{CH}$ vibrations $v_{2}$ and $\nu_{9}$ on a torsional rotation of $2 \pi$. Accordingly, they solved the torsional motion with $4 \pi$ boundary conditions. Wang and Perry $^{9}$ found that an internal coordinate model of the $\mathrm{CH}$ stretches with only Jahn-Teller-like coupling terms in $\cos \gamma$ gave the correct tunneling splittings. Clasp and Perry ${ }^{6}$ showed that an adiabatic approximation to this model also gave qualitatively correct results for the tunneling splittings in the first

a) Author to whom correspondence should be addressed. Electronic mail: dperry@uakron.edu excited CH stretch states. Recently, Xu et al. ${ }^{10,11}$ have performed $a b$ initio frequency calculations along the steepest descent internal rotation path. To describe these results, they developed a two-state model in which the E-type symmetric rotor $\mathrm{CH}$ stretch basis states are coupled by both JahnTeller-like $(\exp (i \gamma))$ and Renner-Teller-like $(\exp (-2 i \gamma))$ coupling terms to form the two asymmetric $\mathrm{CH}$ stretch states. $\mathrm{Xu}$ et al. ${ }^{11}$ found that the vibrational amplitudes for both $v_{2}$ and $v_{9}$ returned to their original values (did not change sign) upon a $2 \pi$ internal rotation along the minimum energy path (MEP); that is, the geometric phase accumulated is +1 .

Zwanziger and Grant ${ }^{12}$ studied $E \otimes e$ systems in which both Jahn-Teller and Renner-Teller couplings are present, the former scaling linearly with the deviation $\rho$ from the $C_{3 \mathrm{v}}$ reference geometry and the latter scaling quadratically. They showed that there are necessarily four CI's between the coupled electronic surfaces, one at the $C_{3 \mathrm{v}}$ reference geometry and three more at distorted geometries of $C_{\mathrm{s}}$ symmetry at the values of $\rho$ where the magnitudes of the linear and quadratic couplings become equal. Transport along a path in the twodimensional $(\gamma, \rho)$ coordinate space that encloses one CI results in the accumulation by the electronic wavefunctions of a geometric phase of -1 and that a path enclosing all four CI's results in a geometric phase of $(-1)^{4}=+1$.

While their work $^{12}$ was formulated in the context of a doubly degenerate $(E)$ electronic state interacting with a degenerate $(e)$ vibrational mode, the same formalism may also be applied to the case of methanol. In the paragraphs below, we present the first example of a set of vibrational CI's in an $E \otimes e$ system. In methanol, the adiabatic separation is between the high-frequency degenerate $\mathrm{CH}$ stretch in the electronic ground state and a pair of large-amplitude low-frequency modes. The energies of the asymmetric $\mathrm{CH}$ stretch vibrations are considered as functions of the torsional angle, $\gamma$, and the $\mathrm{COH}$ bend angle, $\rho$. In the $C_{3 \mathrm{v}}$ reference geometry where the $\mathrm{COH}$ group is linear $\left(\rho=0^{\circ}\right)$, the two asymmetric $\mathrm{CH}$ stretch vibrations ( $v_{2}$ and $\left.v_{9}\right)$ become degenerate $(E)$, and the large-amplitude coordinates $\rho$ 

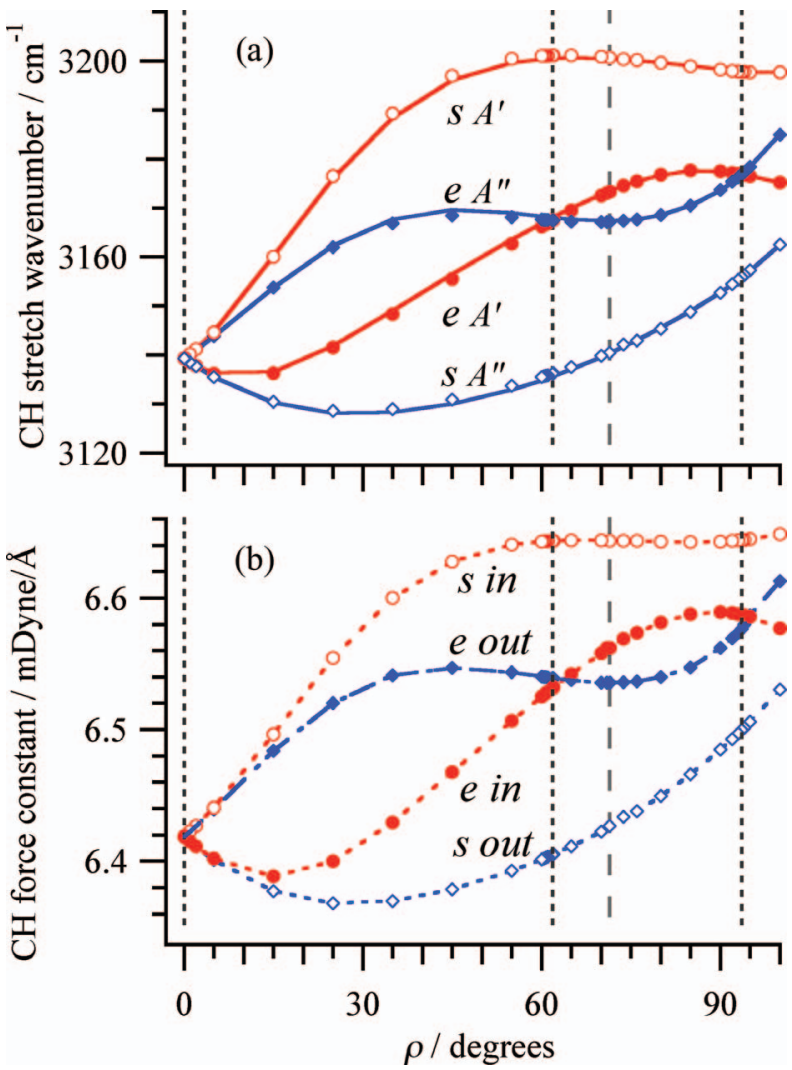

FIG. 1. (a) Vibrational frequencies for the two asymmetric $\mathrm{CH}$ stretch vibrations $\left(v_{2} \mathrm{~A}^{\prime}\right.$ and $\left.v_{9} \mathrm{~A}^{\prime \prime}\right)$ of methanol computed at the $a b$ initio level MP2/6$311+\mathrm{G}(3 \mathrm{df}, 2 \mathrm{p})$ for conformations of $C_{s}$ symmetry. The abscissa is the $\mathrm{COH}$ bend angle $\rho$, measured relative to linearity $\left(\rho=0^{\circ}\right)$. The staggered $(s)$ and eclipsed $(e)$ conformations are indicated. The model calculation is shown as solid lines. The vertical lines, extending through both parts of the figure, indicate the $\rho$ angles at which the $\mathrm{A}^{\prime}$ and $\mathrm{A}^{\prime \prime}$ frequencies cross (short dashes) and also the equilibrium geometry (long dashes). (b) The harmonic force constants for individual $\mathrm{CH}$ bonds in the $C_{s}$ plane of symmetry (in) and outof-plane (out) cross at nearly the same $\rho$ angles.

and $\gamma$ together become a degenerate $\mathrm{COH}$ bending coordinate (e).

One significant difference encountered when applying the $E \otimes e$ formalism to the vibrationally adiabatic surfaces in methanol is that the equilibrium geometry is now far from the $C_{3 \mathrm{v}}$ reference geometry $\left(\rho=71^{\circ}\right)$ rather than close to it as is typically the case for Jahn-Teller coupling between electronic surfaces. Whereas Zwanziger and Grant ${ }^{12}$ neglected anharmonicity and kept only the coupling terms of the lowest order in $\rho,\left(\rho \exp (i \gamma)\right.$ and $\left.\rho^{2} \exp (-2 i \gamma)\right)$, it will be necessary in the methanol case to include higher-order terms in $\rho$.

To search for CI's in methanol, $a b$ initio calculations were undertaken for $C_{s}$ symmetry conformations, both staggered $\left(\gamma=180^{\circ}\right)$ and eclipsed $\left(\gamma=0^{\circ}\right)$ using the same level of theory employed by $\mathrm{Xu}$ et al. $^{11,13}$ ((MP2 = Full/6$311+\mathrm{G}(3 \mathrm{df}, 2 \mathrm{p})$, OPT $=(\mathrm{Z}$-matrix,Vtight $))$. The stationary points on the electronic potential energy surface are the global minima $\left(\rho=71.42^{\circ}, \gamma=60^{\circ}, 180^{\circ}, 300^{\circ}\right)$, the torsional saddle points $\left(\rho=70.95^{\circ}, \gamma=0^{\circ}, 120^{\circ}, 240^{\circ}\right)$, and the $C_{3 \mathrm{v}}$ symmetry point $\left(\rho=0^{\circ}\right)$. Away from these stationary points, partially optimized calculations were done with $\rho$ and $\gamma$ fixed at particular values and the other 10 internal coor- dinates optimized to obtain the lowest electronic energy. In methanol, vibrational CI's occur when the two asymmetric $\mathrm{CH}$ stretch vibrations, $\mathrm{A}^{\prime} v_{2}$ and $\mathrm{A}^{\prime \prime} v_{9}$, become degenerate. Vibrational frequency calculations at each of the computed $C_{s}$ geometries (Fig. 1) show that the $\mathrm{A}^{\prime}$ and $\mathrm{A}^{\prime \prime}$ frequencies, degenerate by symmetry at $\rho=0^{\circ}$, also cross in eclipsed methanol at $\rho=61.9^{\circ}$ and $93.6^{\circ}$. In addition, 95 $a b$ initio points in non- $C_{s}$ geometries were computed in the vicinities of the crossings, along the torsional MEP, and at $\gamma=30^{\circ}$ to make a total of 159 unique $a b$ initio points (see Tables S1 and S2 and Fig. S1 in the supplementary material $\left.^{14}\right)$.

There are a number of conceptual steps to identify the crossing of these frequencies and force constants with CI's. Within the harmonic approximation used in the normal mode analysis, the $\mathrm{CH}$ stretch frequencies provide approximations to the energies of the quantum mechanical $\mathrm{CH}$ stretch excited states relative to the zero-point vibrational level. Since these vibrational energies are calculated for the high-frequency $\mathrm{CH}$ stretch modes as a function of the large-amplitude coordinates $\rho$ and $\gamma$, they represent an adiabatic separation of the highand low-frequency vibrational modes.

Strictly speaking, the normal mode calculation is only valid at the stationary points; however, we argue that the $\mathrm{CH}$ stretches mix only negligibly with the low frequency modes and that the computed "normal mode" frequencies provide a credible description of how the $\mathrm{CH}$ frequencies vary as a function of the low-frequency coordinates. Evidence to support this assertion comes from the fact that the crossings of the normal mode frequencies (Fig. 1(a)) occur at nearly the same bending angles as the crossings of the single $\mathrm{CH}$ bond force constants (Fig. 1(b)). The overall appearance of the graphs in Figs. 1(a) and 1(b) is very similar suggesting that the existence and locations of the frequency crossings are primarily attributable to the variation of the single-bond force constants. Additional evidence supporting the validity of the $\mathrm{CH}$ stretch normal mode frequencies computed at non-stationary points comes from the close agreement of projected and nonprojected frequency calculations along the torsional MEP (see Fig. S2 in the supplementary material ${ }^{14}$ ).

Calculations at the lower level, B3LYP//6-31+G(2d,p), yielded frequency crossings in the same locations $\left( \pm 0.5^{\circ}\right)$ and the resulting graphs (see Fig. S3 in the supplementary material ${ }^{14}$ ) are essentially identical in appearance to Fig. 1. In new report, ${ }^{15}$ higher level calculations (CCSD(T)/augccpVTZ) confirm the MP2 results of Xu et al., ${ }^{11}$ regarding the behavior along the torsional MEP. The consistency across different levels suggests that neither the existence nor the locations of the CI's is strongly dependent on the level of calculation.

With 5 of the 12 internal coordinates, the $3 \mathrm{CH}$ stretches, $\rho$ and $\gamma$, treated explicitly, the other 7 vibrational coordinates are only represented implicitly by their variation in the partially optimized calculations. The relative energies of the different CI's are given by the variation of the energies of the $\mathrm{CH}$-stretch states added to the much larger variation of the electronic potential. For example, at the present MP2 level, the torsional saddle points are $357 \mathrm{~cm}^{-1}$ above the global minima and the $\mathrm{C}_{3 \mathrm{v}}$ symmetry point at $\rho=0^{\circ}$ is at $10967 \mathrm{~cm}^{-1}$. 
Following Zwanziger and Grant, ${ }^{12}$ we apply an adiabatic 2-state model for the asymmetric $\mathrm{CH}$ stretch frequencies,

$$
\begin{aligned}
& \frac{H_{v i b}^{\text {adiabatic }}}{h c} \\
& \quad=\left[\begin{array}{cc}
\omega_{0}(\rho) & \frac{1}{2} f(\rho) e^{-i \gamma}+\frac{1}{2} g(\rho) e^{2 i \gamma} \\
\frac{1}{2} f(\rho) e^{i \gamma}+\frac{1}{2} g(\rho) e^{-2 i \gamma} & \omega_{0}(\rho)
\end{array}\right]
\end{aligned}
$$

but here allowing higher-order terms in $\rho$ with the polynomial expansions,

$$
\begin{aligned}
\omega_{0}(\rho) & =\omega_{0}^{(0)}+\sum_{i=2}^{7} \omega_{0}^{(i)} \rho^{i}, \\
f(\rho) & =\sum_{i=1}^{7} f^{(i)} \rho^{i}, \\
g(\rho) & =\sum_{i=2}^{7} g^{(i)} \rho^{i} .
\end{aligned}
$$

The eigenvalues of the Hamiltonian in (1) are

$$
\begin{aligned}
& \omega_{ \pm}^{\text {adiabatic }}(\gamma, \rho) \\
& \quad=\omega_{0}(\rho) \pm \frac{1}{2}\left[f^{2}(\rho)+2 f(\rho) g(\rho) \cos 3 \gamma+g^{2}(\rho)\right]^{\frac{1}{2}} .
\end{aligned}
$$

Equation (3) was fitted to the $C_{s}$ symmetry data (Fig. 1(a)), including both the staggered and eclipsed conformations. The $\rho$-dependent parameters, Eq. (2), are plotted in Fig. 2, and the resulting adiabatic surfaces are plotted in Fig. 3. Although the fit included only data from the $C_{s}$ geometries, the resulting surfaces also provide satisfying agreement with all of the non- $C_{s}$ data as well. The overall root-meansquare deviation of the model compared to all 318 ab initio frequencies is $0.9 \mathrm{~cm}^{-1}$.

Most prominent in Fig. 3 is the presence of seven conical intersections, one occurring in the $C_{3 \mathrm{v}}$ reference geometry ( $\rho$ $\left.=0^{\circ}\right)$, and six more occurring in eclipsed conformations $\left(C_{s}\right)$

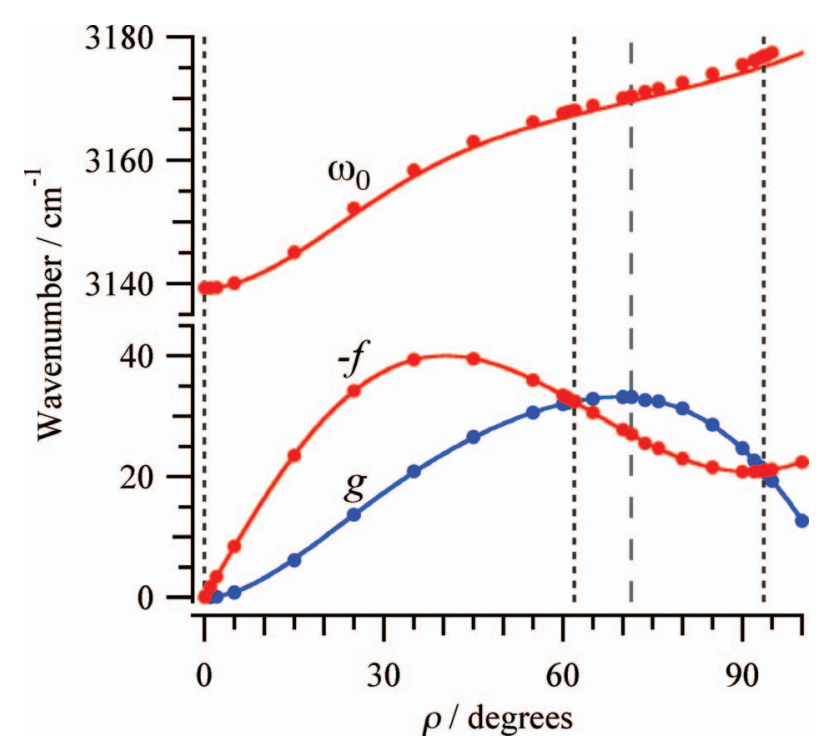

FIG. 2. The model parameters obtained from the overall fit (lines) and computed independently at each value of $\rho$ (points). The vertical guide lines are the same as in Fig. 1.

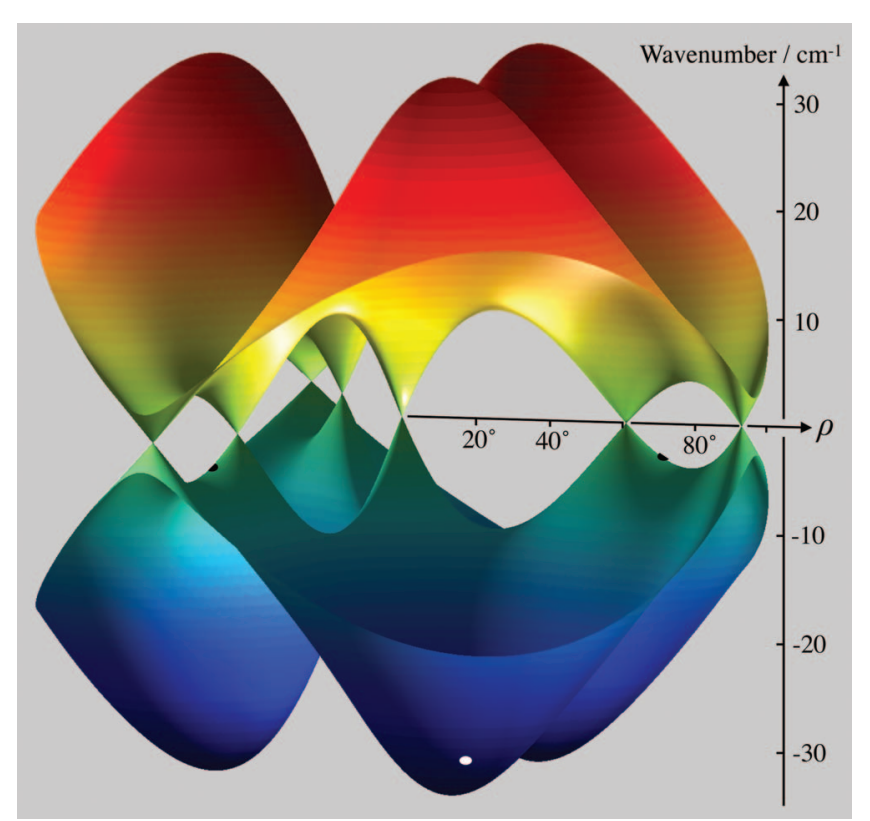

FIG. 3. Relative model frequencies of the two asymmetric $\mathrm{CH}$ stretch vibrations in methanol, represented as surfaces in the two-dimensional coordinate space of the $\mathrm{COH}$ bend angle $\rho$ and the torsional angle $\gamma$. The figure shows seven conical intersections: one at $\rho=0^{\circ}$, three at $\rho=62^{\circ}$, and three at $\rho=94^{\circ}$.

at $\rho=62^{\circ}$ and $94^{\circ}$. The Cl's occur when the off-diagonal terms in Eq. (1) are zero. In the present situation, this occurs in the eclipsed conformation when the $g$ and $f$ parameters are equal in magnitude (Fig. 2). Since the CI's at $62^{\circ}$ are close to the respective torsional saddle points at $\rho=71^{\circ}$, that is, within the range of the $\mathrm{COH}$ zero-point bending amplitude, these CI's are accessible at relatively low energies and therefore may impact the molecular dynamics under a wide range of conditions.

To model their ab initio frequencies along the torsional MEP, Xu et al. ${ }^{11}$ developed a model (referenced here as the XHL model) with two coupling parameters, $k_{1}$ and $k_{2}$,

$$
\omega_{ \pm}^{2}(\gamma)=\frac{1}{m}\left\{k_{E} \pm\left[k_{1}^{2}+k_{2}^{2}+2 k_{1} k_{2} \cos 3 \gamma\right]^{\frac{1}{2}}\right\} .
$$

Using the approximation $(1+x)^{1 / 2} \approx 1+x / 2$ for small $x$, the XHL model is equivalent to the present model (Eq. (3)) at a fixed value of $\rho$, with $\omega_{0}^{2}=k_{E} / m, f^{2}=k_{1}^{2} / m k_{E}$, and $g^{2}=k_{2}^{2} / m k_{E}$. In this context, the present model can be thought of as an extension of the XHL model to include the $\rho$-dependence of their parameters.

To this point, we have considered two low-frequency, large-amplitude coordinates, $\rho$ and $\gamma$. When the dimensionality of the low-frequency coordinate space is 3 or more, the CI's become seams or hyper-seams of CI's. ${ }^{16-18}$ All eight of the low-frequency modes, including the methyl rocks and deformations, could arguably be included in the lowfrequency coordinate space. At present, for purpose of exemplifying seams of conical intersections, we choose to include just one additional coordinate, the $\mathrm{CO}$ stretch $r_{\mathrm{CO}}$, to form a three-dimensional low-frequency coordinate space. In $\mathrm{CH}_{3} \mathrm{OH}$, the $\mathrm{CO}$ stretch is the second lowest vibration after the torsion; in $\mathrm{CH}_{3} \mathrm{OD}$, the torsion, $\mathrm{COD}$ bend, and $\mathrm{CO}$ stretch 


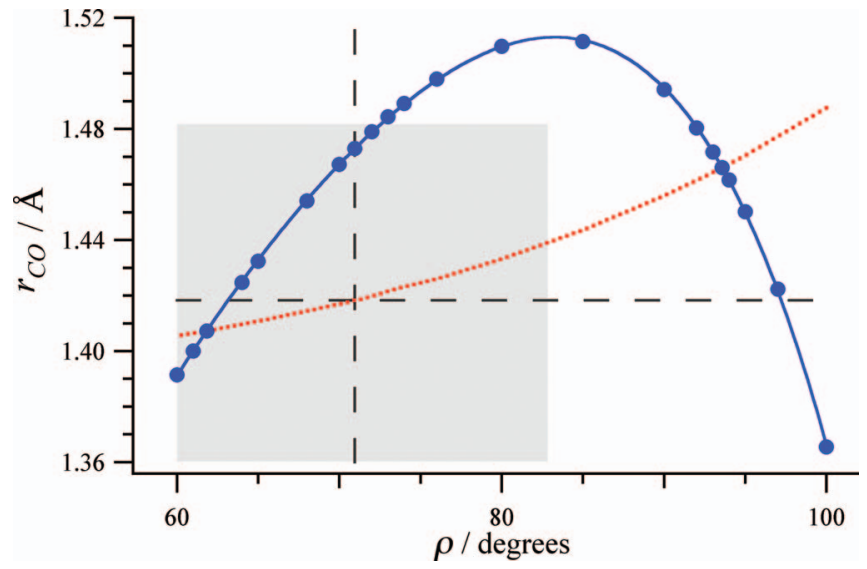

FIG. 4. The location of the seam of conical intersections for eclipsed methanol (markers) and a polynomial fit (solid blue line) is plotted in terms of the $\mathrm{CO}$ bond length and the $\mathrm{COH}$ bending angle. The red dotted line represents the partially optimized calculations of Fig. 1 and the conical intersections shown in Figs. 1 and 3 are located at the intersections of the blue solid and red dotted lines. The dashed lines indicate the zero-point geometries of both coordinates, and the grey shaded area represents the approximate extent of the zero-point amplitudes.

are the three lowest frequencies. ${ }^{19}$ Proceeding with the above methodology, we varied $\rho$ at several different fixed values of $r_{\mathrm{CO}}$ to find a seam of CI's (Fig. 4) in the eclipsed plane.

Fig. 4 shows that the CI's at $\rho=62^{\circ}$ and $94^{\circ}$ belong to the same seam of CI's, a significant length of which lies within the range of the zero-point motions of the $\mathrm{COH}$ bend and $\mathrm{CO}$ stretch. This reinforces our assertion that the CI's are accessible to the dynamics at relatively low energies. Altogether then in the three-dimensional $\gamma, \rho, r_{\mathrm{CO}}$ space, the data reveal four seams of CI's, one symmetry-required seam along the $\mathrm{C}_{3 \mathrm{v}}$ symmetry line $\rho=0^{\circ}$, and three more symmetryallowed seams like the one represented in Fig. 4, one in each eclipsed plane, $\gamma=0^{\circ}, 120^{\circ}$, and $240^{\circ}$.

These conical intersections illuminate the role of geometric phase in methanol. Since four CI's are enclosed by the MEP for a $2 \pi$ torsional rotation, the Zwanziger and Grant theorem ${ }^{12}$ predicts a geometric phase of +1 in agreement with the findings of $\mathrm{Xu}$ et al. ${ }^{11,15}$ However, from a semiclassical point of view, one might consider additional classical paths for a $2 \pi$ torsional rotation. Since the $\mathrm{COH}$ zero-point bending amplitude is large $\left( \pm 11^{\circ}\right)$ extending through the location of the CI's at $62^{\circ}$, there are other possible torsional paths that could enclose an odd number of CI's, say 1 or 3 . Thus, in a fully coupled treatment, the $v_{2}$ and $v_{9}$ wavefunctions may be linear combinations of basis states of differing geometric phase, with the +1 contribution being dominant. States with mixed geometric phase have been found previously in model calculations on methanol. ${ }^{6,8,9,20}$

Since these CI's exist only as an abstraction in the context of an approximate adiabatic separation of high- and lowfrequency vibrations, it is relevant to ask, what, if any, impact do they have on the observable energy level structure of methanol? Xu et al. ${ }^{11}$ have already shown that physically dif- ferent models ${ }^{7,9,11}$ containing one or both of the Jahn-Tellerand Renner-Teller-like coupling terms are equally good at predicting the inverted torsional tunneling splittings in the $\mathrm{CH}$-stretch fundamentals. However, the CI's will likely impact the energy level patterns of the higher torsional and $\mathrm{COH}$ bending levels built on the $\mathrm{CH}$ stretch fundamentals and overtones.

The CI's have direct implications for both the intramolecular and intermolecular dynamics of methanol. Xu et al. ${ }^{11,15}$ have shown that the vibrational characters of $v_{2}$ and $v_{9}$ change sharply over a small range of the torsional angle near the eclipsed conformation. The presence of nearby CI's explains this behavior and will allow quantitative predictions of nonadiabatic processes (surface hopping) near the eclipsed geometry. In general, in the context of either intramolecular or collision-induced dynamics, the CI's provide a connection between the vibrationally adiabatic surfaces. Therefore, one should expect acceleration of energy transfer processes in localized regions around the CI's. Just as electronic conical intersections are now known to be ubiquitous throughout electronic spectroscopy, ${ }^{1-3}$ vibrational conical intersections may also be widespread, consequently impacting the vibrational dynamics $^{4,5}$ in diverse chemical systems.

The authors are grateful to David Yarkony and Tucker Carrington for helpful discussions. Support for this work was provided by the Division of Chemical Sciences, Offices of Basic Energy Sciences, Office of Energy Research, U.S. Department of Energy under Grant No. DE-FG02-90ER14151.

${ }^{1}$ D. R. Yarkony, Rev. Mod. Phys. 68, 985 (1996).

${ }^{2}$ J. D. Coe and T. J. Matinez, J. Am. Chem. Soc. 127, 4560 (2005).

${ }^{3}$ B. E. Applegate, T. A. Barckholtz, and T. A. Miller, Chem. Soc. Rev. 32, 38 (2003).

${ }^{4}$ P. Hamm and G. Stock, Phys. Rev. Lett. 109, 173201 (2012).

${ }^{5}$ P. Hamm and G. Stock, Mol. Phys. 111, 2046 (2013).

${ }^{6}$ T. N. Clasp and D. S. Perry, J. Chem. Phys. 125, 104313 (2006).

${ }^{7}$ B. Fehrensen, D. Luckhaus, M. Quack, M. Willeke, and T. R. Rizzo, J. Chem. Phys. 119, 5534 (2003).

${ }^{8}$ D. S. Perry, J. Mol. Spectrosc. 257, 1 (2009).

${ }^{9}$ X. Wang and D. S. Perry, J. Chem. Phys. 109, 10795 (1998).

${ }^{10}$ L.-H. Xu, J. T. Hougen, J. M. Fisher, and R. M. Lees, J. Mol. Spectrosc. 260, 88 (2010).

${ }^{11}$ L.-H. Xu, J. T. Hougen, and R. M. Lees, J. Mol. Spectrosc. 293-294, 38 (2013).

${ }^{12}$ J. W. Zwanziger and E. R. Grant, J. Chem. Phys. 87, 2954 (1987).

${ }^{13}$ M. J. Frish, G. W. Trucks, H. B. Schlegel et al., Gaussian 09, Revision B.0.1, Gaussian, Inc., Wallingford, CT, 2009.

${ }^{14}$ See supplementary material at http://dx.doi.org/10.1063/1.4871657 for Tables S1 and S2 and Figures S1-S3 providing further documentation of the computed ab initio points.

${ }^{15}$ L.-H. Xu, R. M. Lees, J. T. Hougen, J. M. Bowman, X. Huang, and S. Carter, J. Mol. Spectrosc. (2014) (published online).

${ }^{16}$ J. Dillon and D. R. Yarkony, J. Phys. Chem. A 117, 7344 (2013).

${ }^{17}$ M. J. Paterson, M. J. Bearpark, M. A. Robb, and L. Blancafort, J. Chem. Phys. 121, 11562 (2004).

${ }^{18}$ M. J. Paterson, M. J. Bearpark, M. A. Robb, L. Blancafort, and G. A. Worth, Phys. Chem. Chem. Phys. 7, 2100 (2005).

${ }^{19}$ E. L. Sibert III and J. Castillo-Chara, J. Chem. Phys. 122, 194306 (2005).

${ }^{20}$ D. S. Perry, J. Phys. Chem. A 112, 215 (2008). 